\title{
La recherche en sciences sociales au Dahomey et au Bénin depuis les années 1970 : L'hégémonie du paradigme développementiste
}

Thomas Bierschenk et Roch L. Mongbo

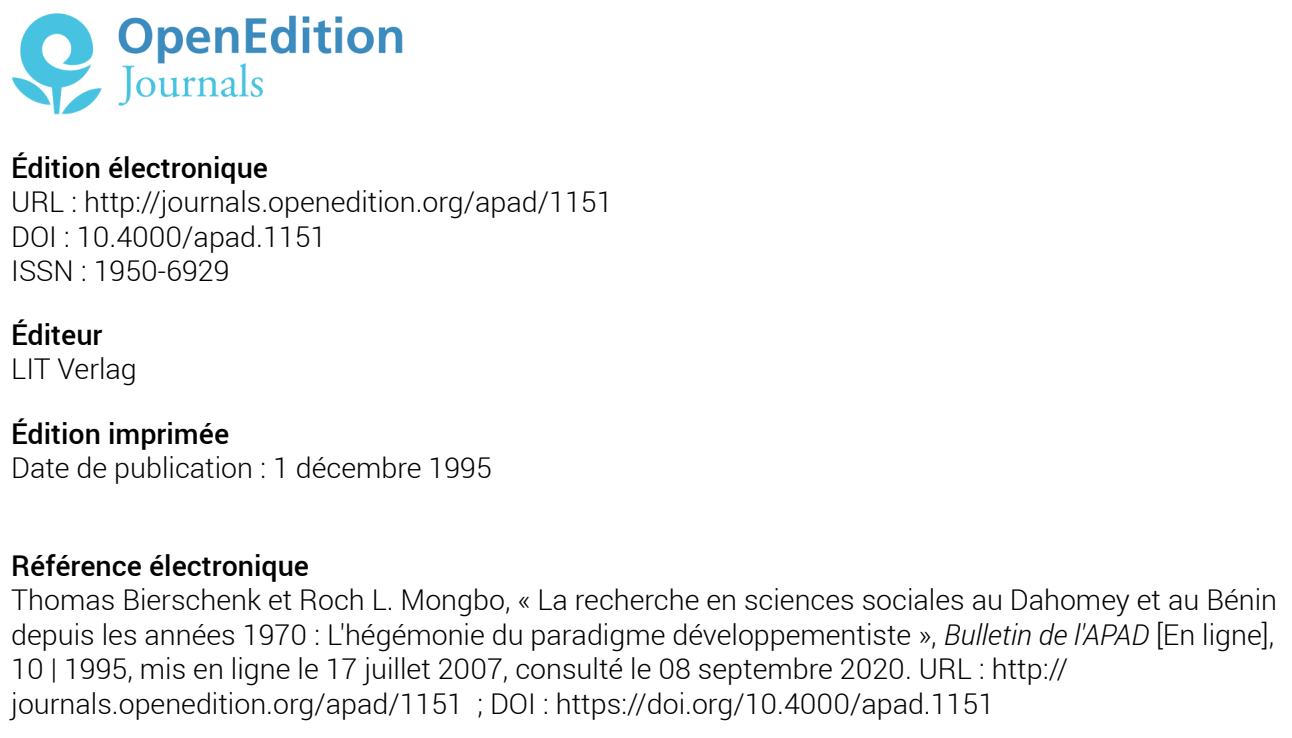

Ce document a été généré automatiquement le 8 septembre 2020

Bulletin de I'APAD 


\title{
La recherche en sciences sociales au Dahomey et au Bénin depuis les années 1970 : L'hégémonie du paradigme développementiste
}

\author{
Thomas Bierschenk et Roch L. Mongbo
}

1 Ce texte était à l'origine prévu comme partie de l'introduction au dossier sur "Le Bénin" qui était piloté par les deux auteurs pour la revue "Politique Africaine" (n59, octobre 1995). Vu l'épaisseur du dossier qui dépassait de loin les normes de la revue, il a été décidé d'enlever cette partie. Comme il nous semble que la situation que nous décrivons pour le Bénin est largement comparable à celle d'autres pays africains, nous reproduisons ce texte ici - dans l'espoir ainsi de motiver une réflexion comparative en matière de sociologie de sciences sociales en Afrique qui nous semble faire largement défaut ${ }^{1}$.

2 Jusque dans les années 1960, l'ensemble du dispositif de la recherche au Dahomey, en sciences sociales ou dans les autres domaines, apparaissait comme un appendice de la machinerie française de recherche; elle se ramifiait en institutions telles que l'IRHO pour le palmier à huile, l'IRAT pour les cultures vivrières, l'IRCT pour le coton, et des instituts non spécialisés comme l'ORSTOM et l'IFAN. Cette situation offrait au moins l'-avantage de permettre au Dahomey de disposer de l'une des meilleures bibliothèques sur le pays au niveau international (celle de l'IFAN à Porto-Novo). Tout chercheur ayant tenté au moins une fois ces dernières années de s'informer sur place sur les recherches en cours au sur le Bénin, a pu mesurer à quel point la situation s'était dramatiquement dégradée. Une véritable politique en matière de recherche faisait défaut, non seulement dans les sciences sociales, mais aussi et surtout concernant une stratégie de formation et de renforcement des capacités nationales de recherche", le tout aggravé par une carence en personnel d'encadrement. Que les Études dahoméennes, qui constituaient un espace d'expression de la recherche dahoméenne en sciences sociales reconnu internationalement, aient disparu dès la fin des années soixante nous parait à cet égard tout à fait symptomatique. 
3 La création de l'Université nationale du Bénin à l'aube des années soixante-dix aurait pu enrayer ce déclin, grâce notamment à ses facultés des sciences sociales, la FLASH (avec ses départements de Lettres, d'Histoire, de Géographie, et de Sociologie \& Philosophie), la FSA (avec le département d'Économie et Sociologie rurales) et la FASJEP. Cependant, même lors de cette phase "révolutionnaire", la France, qui était demeurée l'unique référence en matière de politique de recherche et d'enseignement supérieur, marqua de son empreinte le paysage organisationnel de la recherche scientifique: outre l'université, dont la mission première était l'enseignement, une Direction générale de la recherche scientifique et technique (DGRST) fut créée, sorte de copie simplifiée du CNRS français, au Ministère de l'enseignement supérieur et de la recherche, (on la rebaptisa ensuite Centre Béninois de la Recherche Scientifique et Technique/CBRST) ; parallèlement, le Ministère du développement rural et de l'action coopérative abritait depuis 1984 un département de recherche agronomique (la Direction de recherche agronomique/DRA, aujourd'hui Institut national de recherche agronomique du Bénin/INRAB), doté d'un laboratoire d'Economie et Sociologie rurales. Jusqu'en 1986, la mission centrale de l'université fut de préparer les étudiants à la fonction publique, l'Etat, seul et unique employeur de diplômés universitaires, décidant au niveau central de l'affectation de ces cadres au sein des différents corps administratifs. Un plan de carrière individuel ayant la recherche pour objectif n'avait, compte tenu de cette situation, que fort peu de sens. Plus généralement, le climat politique des années soixante-dix et du début des années quatre-vingt n'était que peu favorable aux chercheurs en sciences sociales. Le régime de Kérékou était peu compréhensif vis-à-vis d'une recherche qui ne se soldait pas immédiatement par l'obtention d'un diplôme. Les contacts avec l'extérieur étaient délicats, en particulier avec la France, tenue responsable du raid des mercenaires de 1977. Un poste au sein d'une organisation internationale était - et reste - préférable à la situation d'un chercheur confronté à des conditions de travail caractérisées par l'isolement et le risque permanents.

4 La recherche en sciences sociales au et sur le Bénin reste jusqu'à nos jours marquée par cette histoire, et ce relativement à trois traits essentiels :

5 1. En premier lieu, le Bénin du point de vue de la connaissance en sciences sociales est pendant longtemps resté quasiment en friche ${ }^{2}$. Cette constatation s'applique à tous les domaines des sciences sociales, de l'anthropologie sociale aux sciences politiques en passant par l'histoire. Il est facile d'illustrer cette situation par un fait concernant les sciences politiques: la publication d'études relatives au politique pris dans son ensemble - aussi bien "par le haut" que "par le bas", ${ }^{3}$ peuvent se compter sur les doigts de la main pour la période qui va des années soixante-dix à la fin des années quatre-vingt - alors que pour la période précédente, il existe un bon nombre d'études solides, y inclus par des auteurs dahoméens ${ }^{4}$. Par contre, les études des années 70 et 80 peu nombreuses ne se fondaient que rarement sur un travail de terrain mais proposaient des interprétations dont la base empirique se réduisait ${ }^{5}$ à des rapports du gouvernement, de la Banque mondiale et des Nations Unies et à des articles de presse.

6 2. Cet exemple illustre bien un deuxième aspect important de la recherche béninoise en sciences sociales : elle est jusqu'à nos jours, au moins en ce qui concerne les textes publiés, essentiellement dominée par les étrangers, même si bien sûr quelques chercheurs béninois en sciences sociales sont reconnus internationalement, comme Paulin Hountondji, John Igué, Félix Iroko, Adrien Whannu, Guy Osito Midiohouan ...). Il 
est intéressant de souligner ici que ces chercheurs étrangers sont souvent non-francophones, et que l'on peut dire qu'un chercheur en sciences sociales pon-béninois s'intéressant au Bénin dans les années quatre-vingt, pouvait tout à fait le faire sans se préoccuper des analyses proposées par ses collègues béninois (il n'y en avait pratiquement aucune!), ou bien sans se donner la peine de coopérer intellectuellement avec les chercheurs nationaux. Les administrations béninoises compétentes pour l'octroi d'autorisation en matière de recherche, n'avaient aucune stratégie identifiable. La partie béninoise ne proposait pas de thème défini de recherche ; aucun intérêt particulier ne se manifestait à pendre part à des discussions sur le contenu des programmes de recherche proposés par les partenaires étrangers; les discussions portaient uniquement sur les conditions matérielles mises à la disposition des chercheurs béninois qui prenaient part aux projets conçus par les chercheurs étrangers. La participation des collègues béninois se réduisait en règle générale à des enquêtes pratiquées dans leurs propres milieux culturels et linguistiques ; ils jouaient ainsi - volontairement - le rôle de simple informateur ${ }^{6}$ (de niveau universitaire). - Et pour les chercheurs étrangers en sciences sociales qui s'intéressaient au Bénin, il était très utile, outre le français, de pouvoir lire également d'autres langues : la maigre littérature publiée à l'aube des années quatre-vingt sur le Bénin le fut souvent en anglais, en allemand et en néerlandais ${ }^{7}$.

7 3. Le rôle dominant de la recherche non-francophone va de pair avec le troisième aspect qui caractérise la recherche béninoise en sciences sociales jusqu'à présent : elle se fait à l'ombre ou dans le sillage des projets et programmes d'aide au développement. En effet, soit elle consiste en une expertise concernant l'avant ou l'après d'un projet de développement, soit les chercheurs participants bénéficient au moins indirectement du dispositif étranger du développement, par exemple du grand nombre de "volontaires" du développement (les allemands à eux seuls en comptent 80 !), dont la présence contribue à faciliter hébergement et transport. Ceci est certainement un facteur non-négligeable qui explique le fort contingent de chercheurs de nationalité allemande et néerlandaise ayant travaillé au Bénin ${ }^{8}$.

8 Cet état des choses se traduisait - et se traduit encore - par une hégémonie remarquable du paradigme du développement sur l'orientation intellectuelle de ces recherches. L'on est tenté de dire que l'Université elle-même doit légitimer son existence en fonction de sa contribution au "développement" national: existe-t-il un rapport de maitrise n'incluant pas un ultime passage relatif aux "conclusions et recommandations" - en substance, si l'on peut dire, "l'Etat doit, l'Etat doit, etc... en matière de développement" - tandis qu'au moins quelques-uns des chercheurs étrangers spécialistes du Bénin, ont apparemment été en mesure, leur carrière avançant, d'échapper tant à la domination du paradigme du développement qu'à celle de la recherche appliquée, et qu'ils ont utilisé leurs expériences de recherche de terrain au Bénin comme fondement à une carrière dans la recherche fondamentale universitaire en sciences sociales.

Un changement de situation a vu le jour à la fin des années quatre :-vingt, sans pourtant remettre fondamentalement en question l'hégémonie du paradigme du développement, ni la domination des chercheurs non béninois. A partir de 1986, le recrutement de tous les diplômés universitaires au sein des services publics fut stoppé, si bien que depuis, chaque année, une cinquantaine de maitrisards en sciences sociales (toutes disciplines confondues) afflue sur le marché du travail. Ces jeunes diplômés sans emploi trouvent pour partie un débouché sur le marché en pleine expansion des 
études de projet. De nombreuses études de faisabilité et de filières ont été engagées en particulier par la Coopération française, le PNUD, la Banque Mondiale, la GTZ, l'UNICEF, etc. dans lesquelles nombre de spécialistes béninois des sciences sociales ont donc été impliqués d'une manière ou d'une autre - le plus souvent, comme simples enquêteurs travaillant avec des questionnaires et répondant aux problématiques et hypothèses élaborées par les agences de développement et leurs experts non-béninois. Dans une large mesure, ce sont ces études qui constituent les références, et sont considérées actuellement comme étant "les recherches en sciences sociales", malgré leur caractère ad hoc, chaotique, résultant des méthodes, d'un montage et d'un planning souvent imposés par des commanditaires à peine au courant des sciences sociales, et débouchant sur des rapports "confidentiels", dont le chercheur n'a pas la propriété, et qu'il est rarement en mesure de capitaliser, et qui de surcroît la plupart du temps disparaissent sous d'épaisses couches de poussière, alors qu'il s'avère essentiel en matière de recherche scientifique de faire l'objet de critiques de la part des pairs. Il convient toutefois ici de comptabiliser positivement la création, au sein de ce marché, de toute une série de laboratoires nationaux et de bureaux d'études privés, dont le plus ancien et le plus crédible semble être le LARES de John Igué, le presque tout dernier en date étant l'Institut de développement et d'échanges endogènes d'Honorat Aguessi.

Toujours est-il, que depuis 1989, le nombre de publications sur le Bénin n'a cessé de s'accroître, et parmi elles, celles produites par des auteurs béninois progressent lentement 9 . Le mérite en revient sans doute au "renouveau démocratique". Sans vouloir sous-estimer la clairvoyance et la justesse des critiques formulées par des nombreux observateurs à l'égard des formes que revêtent la vie politique béninoise, il n'en reste pas moins vrai qu'un dossier tel que celui sur "Le Bénin" (Politique Africaine $\mathrm{n}^{\circ}$ 59) aurait été difficilement concevable dans les années quatre-vingt, à tout le moins avec la participation de chercheurs béninois résidant dans leur pays.

\section{NOTES}

1.Cette genèse du texte explique que les références bibliographiques se limitent au domaine du politique (dans le sens large du terme).

2.Si Christian Coulon (dans Politique Africaine no 45/1992 : 3) considère que le Sénégal serait l'objet d'une "négligence relative" en matière de sciences sociales, alors il conviendrait pour le Bénin de parler "d'ignorance totale".

3.Domaine qui constitue l'essence du dossier sur "Le Bénin" dans Politique Africaine 59 (1995).

4.On ne pensera qu'a : Akindéle \& C. Aguessy, Le Dahomey, Paris 1955 ; Maurice-A. Glélé, Naissance d'un Etat noir. L'Évolution politique et constitutionnelle du Dahomey, Paris 1969 ; Garcia, Luc, L'organisation de l'instruction publique au Dahomey, Cahiers d'études africaines 41/vo1.11 (1971) ; M.A., La république du Dahomey, Paris 1969 ; ;Samuel Decalo, The politics of instability in Dahomey, Acta Africana (Geneve), VII n 2 (1968), 5 - 32 ; Jacques Lombard, La vie politique dans une ancienne société de type feodal, Cahiers d'Études Africaines 1, n 3 (1969), 5 - 45 et les nombreux autres 
travaux de cet auteur; M. Oké, Survivance tribale ou problématique nationale en Afrique ?, Études dahoméennes, N.S. 1, nº 12 (1968), 5-8 ; M. Oké, Des comités électoraux aux partis politiques dahoméens, Revue française d'études politiques africaines $\mathrm{n}^{\circ} 45$, (Sept. 1969), 45 - 61; Dov Ronen, Dahomey. Between Tradition and Modernity, Cornell University Press 1975 ; Staniland, M., The three-party system in Dahomey, 1951 - 1956, Journal of African History 14 (1973) : 291-312 et 491-504 ; Claude Tardits, Porto Novo. Les nouvelles générations africaines entre leurs traditions et l'occident, Paris 1958. 5.Cette tendance est particulièrement nette chez Francine Godin (Bénin 1972 - 1982. La logique de l'État africain, Paris, L'Harmattan 1986), qui considère visiblement les documents utilisés, provenant uniquement de sources administratives, comme la seule réalité significative. L'État béninois (qui du reste apparaît très fortement "anthropomorphisé" dans son livre : on lui impute "un état de conscience claire de son rôle", p. 26) sera par conséquent pris au mot lors de ses communiqués officiels et de ses déclarations d'intention ; l'ouvrage de Godin produit finalement un discours ayant pour objet le discours de la classe politique béninoise de la fin des années 1970, une analyse de son autoportrait et de son auto-représentation. - Les nombreuses publications de Georg Elwert (i.e., Bauem und Staat in Westafrika, Frankfurt, Campus 1983), bien que reposant sur un travail de terrain nettement plus solide, n'échappent pourtant pas complètement à cette tendance "(to describe African politics) as if intentions were reality" (J.-F. Medard, The underdeveloped state in tropical Africa, in : C. Clapham, ed., Private Patronage and Political Power. Political Clientelism in the Modem State, London 1982 : 162)

6.comme l'exprimait le philosophe béninois Paulin Houtondji lors d'un entretien. De nombreux chercheurs en sciences sociales béninois originaires du sud craignent encore aujourd'hui de travailler dans le nord du pays et vice-versa (et ils ne disposent par ailleurs en règle générale pas des compétences linguistiques requises à cet effet). 7.Il convient de noter ici que la littérature en langue allemande, contrairement à celle des collègues néerlandais, n'a été que rarement traduite jusqu'à ce jour ni en français, ni en anglais.

8.Également les enquêtes réalisées par l'un des rares chercheurs nationaux de réputation internationale, John Igué, le furent apparemment dans le contexte d'études sur les courants commerciaux internationaux, et furent financées sur la demande de la CEDEAO et de la CEE (cf entre autres J.O. Igué, "Evolution du commerce clandestin entre le Dahomey et le Nigeria depuis la guerre de Biafra", Canadian Journal of African Studies (1976) : 235-257 ; et "Un aspect des échanges entre le Dahomey et le Nigeria. Le commerce du cacao", Bulletin de l'IFAN, Série B : Sciences Humaines 38 (1976) : 636-669. 9.Sans prétendre à l'exhaustivité, et en nous limitant volontairement aux études relatives au politique, nous renvoyons le lecteur aux textes suivants : C. Allen, "Goodbye to ail that" : The Short and Sad Story of Socialism in Benin", The Journal of Communist Studies 8 (1992) : 63-81; C. Allen, "Restructuring an Authoritarian State : "Democratic Renewal" in Benin", Review of African Political Economy 30 (1992) : 42-58; T. Bierschenk, "The Creation of a Tradition ; Fulani Chiefs in Dahomey/Benin from the Late 19th Century", Paideuma 39 (1993) : 217-244 ; T. Bierschenk, "Demokratische Erneuerung" und "Politik von unten" in der Republik Benin" in : A.-M. Brandstetter et al. (eds.), Afrika hilft sich selbst. Prozesse und Insritutionen der Selbsrorganisation, Münster, Lit- Verlag : 177-187 ; T. Bierschenk, Aussenabhlingigkeir und Intermediarität. Merkmale des Staates in Benin vor 1989 (Sozialanthropologische Arbeitspapiere 52), Berlin, Das Arabische Buch 1993 ; J.R.V. Daane \& R. Mongbo, Peasant influence on development 
projects in Benin : A critical analysis. Genève-Afrique 1992 ; J. Heilbrunn, "Social Origins of National Conferences in Benin and Togo", The Journal of Modem African Studies 31 (1993) : 277-299 ; J. Igué \& B.G.Soulé, l'Etat-entrepôt au Bénin, Paris, Kanhala 1992 ; D. Kohnert \& H. J. Preuss, "Vom Camarade zum Monsieur : Strukturanpassung und Demokratisierung in Benin", Peripherie 46 (1992) : 47-70 ; K. Nwajiaku, "The National Conferences in Benin and Togo Revisited", The Journal of Modem African studies 32 (1994) : 429-447 ; Lazare Séhouéto, "Kulturelle Aktivitiiten Jugendlicher als trojanisches pferd der Modernisierung des Politischen in Westafrika. Zur Vorgeschichte der Demokratisierungsprozesse", in : Brandsteuer op. cit : 29-41 ; E.K. Tall, "De la démocratie et des cultes vodun au Bénin", Cahiers d'Etudes Africaines 137 XXXV (1995) : 195-208; T.Vittin, "Bénin : Du système Kérékou au renouveau démocratique" in : J.-F.Médard (dir.), Etats d'Afrique Noire, Paris, Karthala : 93-115 ; T. Viuin, "Crise, renouveau démocratique et mutations du paysage médiatique au Bénin", Afrique 2000 n 9 (mai 1992) : 37-57.

\section{AUTEURS}

\section{THOMAS BIERSCHENK}

Universität Hohenheim, (490 D) Fruwirsthstrasse 12, D-70593 Stuttgart, Allemagne.

ROCH L. MONGBO

UNB-FSA, BP 526, Cotonou, Bénin. 